\title{
Solar Dynamics Observatory Guidance, Navigation, and Control System Overview
}

\author{
Wendy M. Morgenstern, Kristin L. Bourkland, Oscar C. Hsu, Kuo-Chia Liu, Paul A. C. Mason, \\ James R. O'Donnell Jr., Angela M. Russo, Scott R. Starin, and Melissa F. Vess \\ NASA Goddard Space Flight Center Code 591, Greenbelt, Maryland 20771
}

\begin{abstract}
The Solar Dynamics Observatory (SDO) was designed and built at the Goddard Space Flight Center, launched from Cape Canaveral on February 11, 2010, and reached its final geosynchronous science orbit on March 16, 2010. The purpose of SDO is to observe the Sun and continuously relay data to a dedicated ground station. SDO remains Sun-pointing throughout most of its mission for the instruments to take measurements of the Sun. The SDO attitude control system (ACS) is a single-fault tolerant design. Its fully redundant attitude sensor complement includes sixten coarse Sun sensors (CSSS), a digita Sun sensor (DSS), three two-axis inertial reference units (IRUs), and two star trackers (STs). The ACS also makes use of the four guide telescopes included as a part of one of the science instruments. Attitude actuation is performed using four reaction whels assemblies (RWAs) and eight thrusters, with a single main engine used to provide velocity-change thrust for orbit raising. The attitude control software has five nominal control modes, thre whel-based modes and two thruster-based modes. A whel-based Safehold running in the attitude control electronics box improves the robustness of the system as a whole. All six modes are designed on the same basic proportiona-integral-derivative attitude error structure, with more robust modes setting their integral gains to zero. This paper details the final overall design of the SDO guidance, navigation, and control (GN\&C) system and how it was used in practice during SDO launch, commissioning, and nominal operations. This overview will include the ACS control modes, attitude determination and sensor calibration, the high gain antenna (HGA) calibration, and jitter mitigation operation.

The Solar Dynamics Observatory mission is part of the NASA Living With a Star program, which seeks to understand the changing Sun and its effects on the Solar System, life, and society. To this end, the SDO spacecraft carries thre Sun-observing instruments: Helioseismic and Magnetic Imager (HMI), led by Stanford University; Atmospheric Imaging Assembly (A|A), led by Lockhed Martin Space and Astrophysics Laboratory; and Extreme Ultraviolet Variability Experiment (EVE), led by the University of Colorado. The basic mission is to observe the Sun for a very high percentage of the 5-year mission (10-year goa) with long stretches of uninterrupted observations and with constant, high-datarate transmission to a dedicated ground station to be located in White Sands, New Mexico. These goals guided the design of the spacecraft bus that will carry and service the three-instrument payload. Overarching design goals for the bus are geosynchronous orbit, near-constant Sun observations with the ability to fly through eclipses, and constant HGA contact with the dedicated ground station. A three-axis stabilized ACS is needed both to point at the Sun accurately and to keep the roll about the Sun vector correctly positioned with respect to the solar north pole. This roll control is especially important for the magnetic field imaging of HMI.

The mission requirements have several general impacts on the ACS design. Both the AIA and HMI instruments are very sensitive to the blurring caused by jitter. Each has an image stabilization system (ISS) with some ability to filter out high frequency motion, but below the bandwidth of the ISS the control system must compensate for disturbances within the ACS bandwidth or avoid exciting jitter at higher frequencies.

Within the ACS bandwidth, the control requirement imposed by AIA is to place the center of the solar disk no more than 2 arcsec, $3 \sigma$, from a body-defined target based on one of the GTs that accompany the instrument. This body-defined target, called the science reference boresight (SRB), was determined from the postlaunch orientation of the GTs by averaging the bounding telescope boresights for pitch to get a pitch SRB coordinate, and by averaging the bounding boresights for yaw to get the yaw SRB coordinate. The location of this SRB in the 0.5-deg field-ofview for each GT then becomes the central target for each telescope; one GT is selected for use as the ACS controlling guide telescope (CGT) at any given time. Fine Sun-pointing is effected based on this SRB for all three instruments when the Sun is within the linear range of the CGT.

In addition to limiting jitter, HMI science requires averaging several observations, making the instrument sensitive to low frequency motion that induces differential motion between each observation. This requires the spacecraft attitude to be stable about the roll axis to approximately 10 arcsec over a ten-minute period. Instrument calibrations require that the spacecraft point the SRB up to 2.5 degrees in pitch and yaw away from the center of the Sun, placing the Sun outside the field-of-view of the guide telescopes. In such instances, when the GTs cannot
\end{abstract}


provide the definitive target for the ACS, on-board attitude determination combined with ephemeris prediction of the Sun direction must provide the definitive target.

EVE is capable of observing the Sun with less dependence on attitude control. However, the ground data processing needs for calibrations result in the most strict attitude knowledge requirements for the mission: $[35,70,70]$ arcsec, $3 \sigma$, of knowledge with respect to the center of the solar disk. In addition to driving the ACS sensor selection, the knowledge requirements, which have their effect primarily during Inertial mode calibrations, drive the accuracy requirements for the solar ephemeris.

The need to achieve and maintain geosynchronous orbit (GEO) drove the need for high-efficiency propulsive systems and appropriate attitude control. The main engine provided high specific impulse for the maneuvers to attain GEO, while the smaller ACS thrusters managed the disturbance torques of the larger engine and provided the capability for much smaller adjustment burns on orbit. SDO's large solar profile means that solar radiation pressure is a large torque disturbance, and the momentum buildup from this disturbance and the GEO altitude drives the ACS to use thrusters to manage vehicle momentum. The demanding data capture budget for the mission, however, requires SDO to avoid frequent thruster maneuvers, while concerns about on-orbit jitter restrict the maximum desired wheel speeds desired from the RWAs. The plan for on-orbit wheel speed and momentum management will be discussed as well as what is now being done in operation after the jitter environment was characterized.

The SDO ACS hardware complement is single-fault tolerant. Two main processors carry virtually identical copies of the command and data handling and ACS software, and two identical attitude control electronics (ACE) boxes carry Coldfire processors with contingency ACS software and other hardware interface cards; the ACE structure allows reaction wheels to be commanded by the Sun-pointing Safehold independent of the Mil Std 1553 data bus. The sixteen Adcole CSSs are grouped into primary and backup sets of eight sensors, each set providing the ability to calculate a sun vector. Each set of eight eyes provides full $4 \pi$-steradian coverage. The Adcole DSS comprises an optics head and a separate electronics box providing a 1553 data interface. The electronics box is mounted inside the Faraday cage created by the spacecraft bus module. The DSS head with its 32- deg square FOV is mounted on the instrument module with its boresight along the spacecraft $X$ axis, nearly aligned with the Sun during observations. Adcole has designed the DSS calibration parameters so that the accuracy is 0.24 arcminutes within 10 deg of the boresight, and diminishes to 3 arcminutes as the Sun moves towards the edges of its FOV. This DSS calibration scheme provides higher accuracy attitude determination over the range of the instrument calibration maneuvers. The three IRUs are TARA-1T units provided by Kearfott, each sensing rate in two orthogonal directions with an accuracy of $0.5 \mathrm{arcsec} / \mathrm{bit}$. With thre units, the rate sensing capability of the IRUs is single-fault tolerant, and the failure detection and correction (FDC) system is capable of reconfiguring rate selection autonomously in the event of an IRU failure. The STs are 2 A-STR autonomous star trackers provided by Galileo Avionica They are positioned so that their boresights are 76 deg apart and approximately parallel to the YZ plane. In nominal Sunpointing attitude, bright light sources are well outside the 55-deg Sun exclusion zone provided by the A-STR shade. Additionally, this configuration provides all necessary attitude knowledge and redundancy about the $X$ axis and combines with the DSS to provide adequate knowledge and redundancy for the $Y$ and $Z$ axes as well. Each A-STR provides 1553 quaternion output with an accuracy of 90 arcsec about the boresight and 30 arcsec about the transverse axes. Four Goodrich E-Wheels serve as the usual attitude control and momentum management actuators. They are arranged in a symmetric, pyramidal configuration about the $X$ axis, with spin axes forming 30 -deg angles with the YZ plane of the spacecraft. At nominal $28 \mathrm{~V}$ bus inputs, the momentum capacity for each RWA is $70 \mathrm{Nms}$, and torque capability is $0.25 \mathrm{Nm}$. However, due to operational constraints, the maximum commanded torque is limited by software to $0.2 \mathrm{Nm}$. Eight attitude control thrusters are designed and built by AMPAC-ISP, and they are placed in four pairs at the aft corners of the vehicle to provide attitude control redundancy. To achieve disturbance rejection in roll, the thrusters are canted inward about the $Z$ axis at an angle of $10 \mathrm{deg}$ to the $X Y$ plane. The primary thrusters are commanded in pairs to effectuate $X-, Y$ - and Z-axis control torques. The thrust provided by each unit is nominaly $5 \mathrm{lbf}$. These thrusters are placed and sized to balance any disturbance torques, especially those from firing the main engine, which is an Aerojet R-4D model bi-propellant engine producing $110 \mathrm{lbf}$ of thrust. 